\title{
Evidence for the BUAS test ability to diagnose Lumbar Radicular-Pain in Low Back Pain patients.
}

\author{
Boaz Samolsky-Dekel ${ }^{1}$, Maria Sorella ${ }^{2}$, Alessio Vasarri ${ }^{2}$, and Rita Melotti ${ }^{1}$ \\ ${ }^{1}$ University of Bologna \\ ${ }^{2}$ Azienda Ospedaliera-Universitaria di Bologna Policlinico S. Orsola-Malpighi, Anesthesia \\ and Pain Therapy Unit, Via Massarenti n. 9, 40138, Bologna, Italy
}

May 6, 2020

\begin{abstract}
Background. Differential diagnosis of Low-back pain (LBP) is complex and a prominent health care issue at all Health-care levels; guidance may come from patients' history cues and clinical examination signs. Human and animal studies report that lumbar radicular pain (LRP) may be diagnosed, at all care settings, by the evaluation of subjective responses of injured lumbar nerves to a strain applied at the buttock. The Buttock Applied Strain (BUAS-test) may guide the differential diagnosis of LBP. Following an ex-adiuvantibus criterion, clinical improvement of LRP, diagnosed with the BUAS-test and congruently treated, may support this test diagnostic ability. Methods. Among $258 \mathrm{LRP}$ patients, positive at V1, to the BUAS-test (with/without positive Straight-Leg-Raising-Test, SLRT), the effect of gabapentin on painDETECT (PD) questionnaire and BPI outcomes was quantified in the follow-up visit (V2). We hypothesized that, at V2, $>50 \%$ of the sample would present negative PD-outcome, significant (t-Test), and ?2 points V2-V1 differences for each the BPI-item's score. Multinomial-Logistic-Regression (MLR) and $\chi 2$ analyses were used to evaluate the PD-V2-outcomes' dependence upon independent variables. Results. Of the sample, $77 \%$ reported Negative PD-V2-outcome. V2-V1 differences of all BPI-items were significant and $>2$ points. PD-V2-outcomes showed significant associations with SLRT-V1 and PD-V1, respectively, but not with gender, age group, or pain-site. MLR showed a significant relationship between SLRT-V1 and PD-V2 outcomes. Conclusions. Among LRP patients, diagnosed by the BUAS-test and treated with gabapentin, all prespecified endpoints were reached. These results may be considered a piece of ex-adiuvantibus evidence for the BUAS-test ability to diagnose LRP. While positive BUAS-test implies potential LRP, the co-presence with positive SLRT may imply a severer LRP condition. Further prospective research, in different settings and direct clinical measures, is needed.
\end{abstract}

\section{Introduction}

Low-back pain (LBP) is complex and a prominent health care issue at all Health-care levels. ${ }^{1}$ Pathophysiology of lower-back and limb pain may include nociceptive back pain, somatic referred pain, radicular pain (RP), and radiculopathy ${ }^{2}$. The latter two are considered neuropathic-pain (NeP) conditions ${ }^{3}$. Radiculopathy is the result of a conduction block along a spinal nerve or its roots while RP is the expression of abnormal neuronal singling arising from an injured dorsal root, its ganglion ${ }^{3}$, or inflamed nerve ${ }^{4-6}$. Nociceptive back pain is due to lumbar-spine nociceptors activation. In contrast, the convergence of activated nociceptive afferents on the spinal-cord second-order neurons which subtend regions of the lower limb cause somatic referred pain. 
Clinically, RP and radiculopathy show neurological signs and symptoms ${ }^{3}$. RP may show dysesthesias, whereas radiculopathy may show paresthesias and negative signs like compromised reflexes, numbness, and weakness depending on whether sensory or motor fibers are involved, respectively.

Differential diagnosis of LBP pain, and hence congruent therapeutic approach, are challenging as clinical presentation patterns are variable and different pathophysiological elements may coexist. A focused history, thorough clinical and neurological examination may guide differential diagnosis and treatment ${ }^{1}$. Anticonvulsants, such as gabapentin or pregabalin, along with antidepressants, are considered the first choice or bridge therapy for non-invasive treatment of RP ${ }^{7-9}$.

We have previously reported the description and reliability features of a clinical adjunctive test, namely, the Buttock Applied Strain (BUAS) test, that may facilitate the differential diagnosis of LBP and may uncover the presence of RP in LBP patients ${ }^{10}$. This test showed high sensitivity, specificity and, linear correlation with the PainDETECT (PD) questionnaire and thus implying the ability to detect the presence of NeP (ie, lumbar-RP) in LBP patients.

To further support the BUAS-test diagnostic ability, we sought to verify the influence of an anticonvulsant (namely, gabapentin) on PD outcomes and pain scores of patients who were positive to the BUAS test and hence diagnosed with potential lumbar-RP. The latter patients are routinely treated in our practice with the gabapentin protocol. We have hypothesized that, given the properties of gabapentin to modulate $\mathrm{NeP}$ (and hence lumbar-RP), a subsequent significant clinical improvement in PD outcomes and pain scores, might be considered a piece ofex-adiuvantibus evidence for the ability of the BUAS test to detect lumbar-RP.

\section{Methods}

\section{Settings and patients}

This retrospective and observational study was held at the acute and CP center of Bologna's Teaching Hospital, Italy. This is an anesthesiology-based pain program that provides outpatient consultation to primary care physicians and specialty services for inpatients. The sample for the present study, according to the inclusion/exclusion criteria (see below), includes $n=258$ consecutive non-cancer LBP outpatients.

\section{Proceedings and instruments}

Routinely, upon first visit (V1) and before the clinical examination, patients complete three questionnaires: Short Portable Mental Status Questionnaire (SPMSQ), the Brief Pain Inventory (BPI), and the painDETECT (PD) questionnaire; BPI and PD are administered to patients also upon follow-ups. Focused clinical history and physical examination for LBP patients include Straight Leg Raising Test (SLRT) and the BUAS test. The rationale, description, and reliability of the BUAS test for detecting lumbar-RP in LBP patients were earlier reported ${ }^{10}$. Briefly, "it consists of identifying, with the patient in the prone position, the Posterior Superior Iliac Spine (PSIS) with the examiner's index or middle finger. Hence, with a full extended hand, the examiner's thumb reaches the greater trochanter; keeping the index or middle finger on the PSIS, the thumb is moved towards halfway between the greater trochanter and the sacrococcygeal symphysis. The thumb thus exerts pressure (ie, the strain) against the underlying bony structures hitting the sciatic nerve. The test is considered positive if the patient reports pain exacerbation different from that of the mere pressure, or reproduction of his/her pain" ${ }^{10}$.

In our practice, all patients, positive to the BUAS test or the SLRT, are prescribed (if no counterindications exist) with gabapentin $300 \mathrm{mg}$ tablets. Our inhouse gabapentin-prescription protocol foresees one tablet at bedtime for three days, hence, one tablet bid (in the morning and at bedtime) for three days, and afterwards one tablet tid. Therapy outcomes are evaluated in the follow-up visits where gabapentin dose may be tapered 
or increased according to the patients' clinical responses. All retrieved clinical and therapy information is stored in the patients' chart and at the clinic's database.

Inclusion criteria for this study were: outpatients with at least one follow-up visit after V1, [?]18 years of age, with chronic LBP ([?]3 months), positive, at V1, for the BUAS test with or without positive SLRT, having had, at the end of V1, gabapentin-protocol prescription, and no pain procedures before the first follow-up visit (V2), and who signed informed consent. Exclusion criteria were: SPMSQ score $<8$, history or diagnosis of cancer or diabetes upon V1 or later, positive clinical signs for the piriformis syndrome, ongoing therapy, at V1, with anticonvulsants (gabapentin or pregabalin) or antidepressants, and finally, withdrawal from the gabapentin protocol, before V2, for severe side effects.

\section{Demographic and clinical predictors}

Demographic predictors are: (I) gender: male/female; (II) age groups [in order to avoid unbalanced over representation in wider age interval groups, patients over 35 years of age are divided into 15-year interval subsets: $36-50 ; 51-65 ; 66-80$ and [?] 81 years (classes B- E, respectively); the only subset having a 17-year interval is that of young adults ie 18-35 years of age (class A)].

Pain-related predictors were retrieved from the BPI. In the latter, a human body image allows the topographical location of the pain site. Pain site categories were: lumbar spine, lumbar spine and sciatica, lumbar spine associated with diffused pain, and lumbar spine associated with other specific pain sites (cervix, groin, dorsal spine, shoulder, and headache). The following five items assess, using a 0-10 numerical rating scale (NRS), the highest, lowest, and mean pain intensity in the past 24 hours, and the actual pain both under static and dynamic conditions. Item- 6 , estimates the percentage of pain relief with the ongoing pain therapy (0\%, no relief $-100 \%$, optimal relief). Finally, items 7-13 assess how much, in the past 24 hours, pain interferes with the patient's QoL (activity in general, mood, ability to walk, work activity, social interaction, sleep, and the enjoyment of life; estimation is made using a 0-10 NRS $(0=$ no interference to $10=$ maximum interference).

The PD is a clinician-administered and patient-reported screening questionnaire to reveal the likelihood of a neuropathic pain component in LBP patients; it consists of seven items that address neuropathic-pain symptoms' quality with a final score between -1 and 38 (a score of [?]12 implies no neuropathic component, a score of [?]19 implies the presence of neuropathic component, while a score of 13-18 implies that the result is uncertain ${ }^{11-13}$.

Finally, based on the results of the SLRT and BUAS test, the sample includes two subsets of patients: those who, at V1, were positive for the BUAS test and negative for the SLRT (BUAS+/SLART-), and those who were positive to both tests (BUAS+/SLART + ).

\section{Ethics}

This study was approved and authorized by the Hospital Ethics Committee (235/2013/O/Oss). The study was conducted according to the Helsinki Declaration and the International Association for the study of Pain (IASP)'s guidelines for pain research in animals and humans. All participants were personally and thoroughly informed by the investigators on the aims of the study. Patients were informed that participation was voluntary, anonymous and would not affect their care; hence, an informed consent was obtained.

\section{Study questions, data presentation and statistical analysis}

Fig. 1 describes the flowchart of the study. We sought to quantify, at V2, the changes in PD outcomes and BPI scores, in patients who were, at V1, positive for the BUAS test, with or without being positive for the SLRT, and thus were prescribed with the gabapentin protocol to treat their potential lumbar-RP condition. We have hypothesized that, at V2, positive BUAS-test patients will report significant improvement in PD 
outcomes and BPI scores. We have established that significant improvement should include the following elements: over $50 \%$ of the sample will present, at V2, negative PD outcome, statistically significant differences of BPI mean-scores between V2 and V1, and that such differences will be [?]2 points for each BPI item. We have established these elements following literature recommendations for achieving a prespecified Minimal Clinically Important Change (MCIC) in pain research ${ }^{14,15}$. We assumed that in case of affirmative results, these might be considered a piece ofex-adiuvantibus evidence for the capacity of the BUAS test to detect lumbar-RP in LBP patients. Finally, we planned to look for associations between patients' independent variables and $\mathrm{PD}$ outcomes at V2 (PD-V2) in order to uncover variables that may undermine the capacity of the BUAS test to detect lumbar-RP.

Continuous data are reported as the mean ( $\pm \mathrm{SD}$, standard deviation); category data are expressed as absolute numbers and percentages. The dependence of PD-V2 outcomes upon independent variable categories was determined using $\chi^{2}$ analysis. Independent variables were gender, age group, pain localization, SLRTV1, and BUAS-test-V1; when significant, a post-hoc cell contribution analysis was performed, and major contributions for the association were reported. Multinomial Logistic Regression (MLR) was used to classify subjects based on a set of predictor variables. Dependent variables for MLR were the PD-V2 outcome classes and the 'negative' class (the most numerous) was the reference outcome class. Independent variables were those used for the above mentioned $\chi^{2}$ analysis. Differences of BPI-items' mean scores between V2 and V1 are reported as absolute difference $(\Delta)$ and $95 \%$ upper and lower confidential intervals $(\mathrm{CI}) ; t$-test was used to compare the BPI-items' scores between V1 and V2. Statistical significance was defined as $\mathrm{p}<0.05$. When appropriate, $\mathrm{p}$ values were rounded to three decimals.

\section{Results}

Table 1 reports the sample and its subsets' demographic and clinical features. The sample included $\mathrm{n}=258$ consecutive cases with mean age of $67.4( \pm 15.0$; range $27-93)$ years; $67.4 \%(\mathrm{n}=174)$ were females. The most frequent pain localizations, $67.3 \%(\mathrm{n}=175)$, was 'lumbar spine and sciatica'. The Median of V1-V2 visit-interval was 82 days. The sample subsets were similar for all demographic and clinical features except for mean age and PD-V1 outcomes. Indeed, the mean age in the BUAS+/SLART+ subset $(64.5 \pm 15.7)$ was slightly lower than that of the BUAS+/SLART- subset $(69.3 \pm 14.3)$; uncertain PD-V1-outcome was reported by $79 \%$ and $19 \%$ of the BUAS+/SLART- and BUAS+/SLART+ subsets, respectively; inverted percentages were reported for the positive PD-V1-outcome. Thirty patients $(1.2 \%)$ reported initial dizziness and somnolence during the gabapentin titration period; however, no patient stopped the therapy as these side effects decreased over time.

\section{PD outcomes}

Table 2 shows the frequency distribution of PD-V1 and PD-V2 outcomes, and the mean $( \pm \mathrm{SD})$ of the PD final scores, split by the two subsets of patients: the BUAS+/SLART-, and the BUAS+/SLART+ one. At V2, $77 \%(\mathrm{n}=199)$ of the entire sample showed negative PD-outcome. In particular, at V2, all patients $(100.0 \%)$ of the BUAS+/SLART- subset showed negative PD-outcome. Of the BUAS+/SLART+ subset, $43 \%$ showed negative PD-outcome at V2. In this subset, positive PD-outcome at V1 decreased at V2 by $60 \%$, and uncertain PD-outcome increased roughly two folds. Finally, in the BUAS+/SLART+ subset, among patients who showed positive PD-outcome at V1,37\% and $23 \%$, respectively, shifted at V2, to uncertain or negative PD-outcomes; $20 \%$ remained, at V2, with positive PD-outcome.

To note, we have compared, both in the sample and the study's subsets, the mean of PD-V1 and PD-V2 final scores (Table 2) and found significant differences between them (t-test, $\mathrm{p}<0.001$, respectively). At V2, there were no PD 'positive' or 'uncertain' outcomes in the BUAS+/SLART- subset. By contrast, in the BUAS+/SLART+ subset, mean PD-V1 final scores (vs PD-V2) decreased, respectively, from 23.8 to 19.0 for the 'positive' PD outcome, and from 18.0 to 15.9 for the 'uncertain' PD outcome. In both subsets, the mean 
of the 'negative' PD-outcome at V2 was 4.3 (BUAS+/SLART-), and 6.7 (BUAS+/SLART+), respectively. Interestingly, in the BUAS+/SLART+ subset, PD-V1 'positive' and 'uncertain' final scores were roughly 5 and 3 points higher.

\section{BPI outcomes}

Fig. 2 shows, for the entire sample, the mean scores of the BPI items, at V1 and V2, and their V2-V1 absolute differences (solid line; V2-V1 values are anchored by a dashed line). For all BPI items, the V2-V1 differences of the BPI item mean scores were of $>2$ points, implying a clinically significant improvement in both pain control and the pain-interference with daily activities. All BPI items showed statistically significant differences between $\mathrm{V} 2$ and $\mathrm{V} 1$ ( $t$-test, $\mathrm{p}<0.001$, respectively). The same results were also found within the study's subsets, respectively. For details view table 3, which shows the mean scores of the BPI items spilt by the BUAS+/SLART- and the BUAS+/SLART+ subsets, their V2-V1 differences ( $\Delta$ and 95\% CI), and the results of the $t$-test analyses. To note, the relief obtained with the therapy improved by roughly $40 \%$ in both subsets. All BPI items in both subsets showed statistically significant differences between V2 and V1 (t-test, $\mathrm{p}<0.001$, respectively).

\section{Associations and MLR}

Table 4 reports the results of the association analyses ( $\chi^{2}$-analysis) between the PD-V2 outcomes and the independent predictors. It also reports the post hoc analyses results as cell contribution for the reported association (the two most influential contributions). No significant associations were found between the PD-V2 outcomes and the independent predictors gender, age groups, and pain sites $\left(\chi^{2}\right.$-analysis, $\left.p>0.05\right)$. Statistically significant associations ( $\chi 2$-analysis, $\mathrm{p}<0.0001$, respectively) were found between the PD-V2 outcomes and the independent predictors SLRT-V1 and PD-V1, respectively. In particular, post hoc analysis showed that the 'positive' and 'negative' SLRT-V1 outcomes were associated, respectively, with 'uncertain' and 'negative' PD-V2 outcomes. Finally, 'positive' and 'uncertain' PD-V1 outcomes were associated, respectively, with 'uncertain' and 'negative' PD-V2 outcomes.

For the MLR analysis, PD-V2 outcomes were the dependent variable (reference outcome class, 'negative') while SLRT-V1 and BUAS-test-V1 outcomes were the predictors. Other predictors were excluded, given the lack of associations observed with the $\chi^{2}$-analysis. As reported in table 5 , The model was found to fit the data significantly $(\mathrm{p}=0.000)$, and the predictor SLRT-V1 had a significant overall effect on the outcome (Likelihood Ratio Tests, $\mathrm{p}=0.000$ ). In particular, for the 'positive' and the 'uncertain' PD-V2 outcomes, the predictor 'negative' SLRT had adjusted odds-ratios ( $\mathrm{p}$-values, $95 \% \mathrm{CI}$ ) of 0.038 , respectively $(\mathrm{p}=0.000,0.012$ 0.115 and 0.016-0.090, respectively). Thus, for 'negative' SLRT cases, the risks for 'positive' or 'uncertain' PD-V2 outcomes to occur decrease versus the Reference outcome class PD-V2 'negative'.

\section{Discussion}

We report the changes in PD and BPI outcomes of $n=258$ LBP patients who were positive to the BUAS test and hence were treated with gabapentin for their potential lumbar-RP condition. After the treatment, all prespecified endpoints of the study were reached. We argue that these results may be considered a piece of ex-adiuvantibus evidence for the ability of the BUAS test to detect lumbar-RP in LBP patients.

In a medical context, the term ex adiuvantibus (from Latin, meaning "from that which helps") refers to the method of making an inference about the presence/cause of a pathological conditionfrom the observed responses of the condition to a giventreatment. In our case, the potential pathological condition was lumbar$\mathrm{RP}$, as the BUAS test potentially diagnosed it.

We have recently proposed the BUAS-test as an adjunctive clinical sign for lumbar-RP ${ }^{10}$. We have argued that positive BUAS test implies potential lumbar-RP, and vice-versa. Lumbar-RP, a known NeP condition, 
can be indirectly quantified by measures like the PD questionnaire and the BPI, which we here used to quantify the observed responses to the given treatment. We hypothesized that a congruent treatment for potential lumbar-RP (in our case, gabapentin) might lead to its clinical improvement. Such improvement is measurable with the tools mentioned above. Thus, subsequent significant improvement in PD and BPI outcomes may imply, ex adiuvantibus, that the underlying pathological condition was indeed lumbar-RP as the BUAS test initially implied it. Accordingly, we argued that should a significant improvement in the lumbar-RP condition occur, it would be reasonable to infer that the BUAS-test did detect the lumbarRP. To further support this inference, we assumed that, after treatment, the observed responses might be considered 'significant improvement' only should these responses fulfill restricted prespecified statistical and clinical endpoints, following the recommendations for achieving MCIC in pain research ${ }^{14,15}$. These endpoints were: over $50 \%$ of the sample should show negative PD-V2 outcome, significant V2-V1 differences of the BPI-items' mean-score, and that these differences should be of at least 2 points for each BPI item.

Lumbar-RP is considered the result of abnormal discharges arising from afferents of an injured dorsal root, ganglion, or inflamed nerve ${ }^{3}$. These abnormal discharges are the expression of nerve roots' sensitization to mechanical stimulation after sustained and protracted compression (i.e., Mass Effect), or their response to the perineural-spread of inflammogenic material arising from the nucleus polposus (i.e., Chemical Radiculitis) 16. These mechanisms induce maladaptive neuronal responses that thus have the potential to generate abnormal responses from the affected nerve ${ }^{17}$. The differential diagnosis of LBP is complicated by the variability and possible coexistence of multiple pathophysiological and presentation patterns. A thorough history and clinical examination, also in primary care settings, are essential in establishing diagnosis and treatment in LBP patients. In particular, symptoms, an accurate neurological examination, imaging, and specific signs (e.g., SLRT and recently, the BUAS test) for lumbar-RP may guide differential diagnosis ${ }^{1}$.

The rationale for the application of the BUAS test is explained elsewhere ${ }^{10}$. Briefly, animal and human research assert that applying a strain on an injured nerve, sensitized or inflamed, may elicit heterospecific discharges, and hence subjective responses in multiple somatosensory afferents. Such elicited responses to the applied strain can confirm the presence of RP in various clinical conditions, including injured/inflamed lumbar dorsal roots and ganglion ${ }^{2,3,5,6,18-25}$.

With the BUAS test, we proposed to apply the strain on the sciatic nerve along its course in the buttock. Several reasons support the feasibility and efficacy of the BUAS test. First, the sciatic nerve, which contains axons from the L4-S3 levels, often involved in lumbar RP, is easily identifiable in the buttock. Second, applying the strain at this proximal sciatic-nerve course, the abnormal responses can be directly associated with proximal nerve injuries. Third, in the buttock, the sciatic nerve lies above stable anatomical structures allowing the strain to be effectively applied. Finally, the BUAS test showed, among LBP patients, satisfactory sensitivity (92\%), specificity (100\%), prior probability, and interrater reliability (Cohen's Kappa, 0.911) ${ }^{10}$. It showed no associations with predictors like gender and age groups while it showed significant associations with predictors like the pain site (lumbar pain and sciatica), and the PD questionnaire. These findings imply that the BUAS test is not compromised by patients' demographic features and supports its content validity. By contrast, the SLRT, widely used for the diagnosis of lumbar-RP, should be considered with caution as it shows crucial limitations ${ }^{26}$. These limitations include the lack of standard procedure for carrying out and interpreting the SLRT, unclear underlying mode-of-action, compromised diagnostic reliability related to specific predictors (the patient's age, gender, psychosocial factors, and diurnal variability), and lack of direct correlation with MRI findings, in case of a herniated disc. Finally, "the diagnostic value of the SLRT in detecting the presence of lumbar disc herniation may lie primarily in ruling out its presence because the test sensitivity (0.8) is far higher than its specificity (0.4)" 26 .

Treating lumbar-RP implies a step-wise approach from diagnosis to treatment (conservative, invasive, or potential surgery) to ameliorate or resolve the lumbar-RP condition and to permit patients to achieve a better functional status and quality of life ${ }^{27}$. Based on moderate or high quality of evidence and efficacy, antidepressants and anticonvulsants (e.g., pregabalin and gabapentin) have strong GRADE recommendations for use in all $\mathrm{NeP}$ conditions and are thus proposed as first-line treatment for $\mathrm{NeP}^{7,28,29}$. Several controlled 
studies have found gabapentinoids to be effective for $\mathrm{NeP}$ and lumbar-RP ${ }^{10,30-34}$. We chose gababapentin both because of our positive experience with this drug, its known pharmacodynamic properties in lumbar$\mathrm{RP}$, and local favorable economic characteristics ${ }^{9}$. Gabapentin is prescribed with a starting dose of $300 \mathrm{mg}$ and may be titrated up to $1800-3600 \mathrm{mg} /$ day with a "low and slow" incremental fashion ${ }^{8,9,35}$. For optimal clinical efficacy gabapentin should be prescribed tidbecause of its short elimination half-life, and saturable absorption system.

Our inhouse protocol for first-line treatment of potential lumbar-RP (i.e., patients positive to the BUAS test and/or the SLRT), is a 7 days titration to $900 \mathrm{mg}$ of gabapentin daily (300 $\mathrm{mg}$ tablets tid). Therapy outcomes and side effects are evaluated in the follow-up visits where doses may be tapered or increased according to the patients' clinical responses. In our sample no patient stopped the therapy because of side effects even though $1.2 \%$ patients reported initial dizziness and somnolence during the gabapentin titration period, which diminished over time.

The observed responses to the gabapentin treatment in this paper were retrieved from the PD and BPI questionnaires. The PD explores the likelihood of the NeP component in LBP patients. The final score of the questionnaire yields the three PD outcomes: negative, uncertain, and positive ${ }^{11-13}$. In our sample, PD-V1 outcomes were positive (45\%) or uncertain (55\%), while PD-V2 outcomes were mostly negative (77\%). These results imply that over $50 \%$ of the sample had a negative PD-V2 outcome and hence fulfilling the first endpoint of the study. Interestingly, while the BUAS+/SLART- subset showed only negative PDV2 outcome, in the BUAS+/SLART+ subset, several PD-V2 outcomes were positive (20\%) or uncertain (37\%). A possible explanation to these results may be that in the BUAS+/SLART+ subset, the underlying lumbar-RP condition was somehow severer. Indeed, unlike the BUAS+/SLART- subset, patients in the BUAS+/SLART + subset were positive to both SLRT and BUAS tests. Further, it is possible that in these patients, the gabapentin dose (300 $\mathrm{mg}$ tid ) was not sufficient to manage the severer lumbar-RP condition. Indeed, recommended daily dose of gabapentin is higher (1800-3600 mg daily) $8,9,35$. Interestingly, we found that in the BUAS+/SLART+ subset, and compared to PD-V1, the mean of the positive PD-V2 final-scores decreased from 23.8 to 19.0 (the lower cutoff of the negative PD outcome) in all patients, and from 18.0 to 15.9 for the uncertain outcome. These results indirectly show that the treatment did affect the severer lumbar-RP condition but yet, it was not clinically sufficient. Congruently, it is reasonable to speculate that the BUAS test may diagnose the presence of potential lumbar-RP also in the absence of positive SLRT. The presence of both signs may imply a severer lumbar-RP condition and possibly the necessity of higher doses of gabapentin or more invasive approach for its treatment. Indeed, in most patients who did not achieve a negative PD-V2 outcome, gabapentin dose was increased, or epidural steroid injections were programmed.

We used the BPI to evaluate changes in patients' pain intensity and its interference with quality of life, given the clinical importance of pain as a primary patient-reported outcome in pain research ${ }^{14}$. This tool permits to follow the key recommendations for reporting pain research outcomes given its established content validity, reliability, and the ability to detect MCIC. In this study, we retrieved information about MCIC from the comparison between V2 and V1 of the BPI items' mean score and their absolute difference. Following literature recommendations for achieving a prespecified MCIC in pain research ${ }^{14,15}$, we have established that for this study the MCIC should include statistically significant differences of BPI mean-scores between V2 and V1 and that such differences will be [?]2 points for each BPI item.

BPI outcomes showed, both in the sample and in the study's subsets, significant V2-V1 differences of the BPI-items' mean-score, and these differences were of [?]2 points for each of the BPI item. These results thus fulfill the second and the third endpoints of the study.

Interestingly, although not significant, V2-V1 absolute differences of the BPI-items' mean-score in the BUAS+/SLART+ subset were slightly higher than in the BUAS+/SLART- subset. In particular, in the BUAS+/SLART+, the BPI pain items (item 1 to 5) showed absolute differences of roughly 3 points or higher; for the QoL items the trend of higher absolute difference was less pronounce but still present. These results indirectly show that the treatment did affect the pain outcomes of the severer lumbar-RP condition, and thus confirming its presence as yielded by the BUAS test. 
Finally, no significant associations were found between the PD-V2 outcomes and the independent predictors gender, age groups, and pain sites. These findings imply that the BUAS test was not compromised by patients' demographic or clinical features and thus supports its reliability and content validity. Significant associations were found between the PD-V2 outcomes and the predictors SLRT-V1 and PD-V1, respectively. In particular, the presence of positive SLRT at V1 was associated with the uncertain PD-V2 outcome while its absence with the negative PD-V2 outcome. These findings imply that, as mentioned above, the co-presence of both signs may denote a severer lumbar-RP condition. In such a condition, the treatment was able to shift part of the positive PD-V1 outcome cases to the uncertain PD-V2 outcome, but not to a negative one like it could in the absence of positive SLRT. Similarly, positive and uncertain PD-V1 outcomes were associated, respectively, with uncertain and negative PD-V2 outcomes. Also, the MLR analysis confirms that for 'negative' SLRT-V1 cases, the risks for 'positive' or 'uncertain' PD-V2 outcomes to occur decrease versus the Reference outcome class PD-V2 'negative'. Albeit, the results mentioned above imply that in the presence of positive BUAS test, with or without positive SLRT, the given treatment (i.e., gabapentin) did affect the lumbar-RP condition.

Study limitations. The main limitation of the study is its observational and retrospective nature. The study's ecological and external validity may be questioned as to the study design of the indirect assessment of the BUAS-test diagnostic ability. Indeed, using the effect of gabapentin on PD and BPI outcomes modifies the direct evaluation method. A direct evaluation would have implied, for example, a comparison of the BUAS test outcomes with invasive neurological tests or imaging studies whose outcomes are not always reliable and conclusive for lumbar-RP diagnosis and often yield ex-adiuvantibus treatments. In this study, data were collected within a setting of routine work. The external validity of such observational study comes from its strong relevance to practice and the ability to highlight meaningful pain assessment solutions.

\section{Conclusions.}

In a sample of LBP patients who were treated with gabapentin because they were diagnosed with potential LRP by the BUAS test, all of the study's prespecified endpoints of MICC in PD and BPI outcome changes were reached. These results may be considered a piece of ex-adiuvantibus evidence for the ability of the BUAS test to detect lumbar-RP. While positive BUAS test implies potential lumbar-RP, the co-presence of positive SLRT, may imply a severer lumbar-RP condition. To generalize findings, more prospective research in different clinical settings and direct clinical measures is needed.

\section{Conflicts of interest}

The Authors declare that there were no funding sources that supported the work and there are no conflicts of interest.

\section{References}

1. Bardin, L. D., King, P. \& Maher, C. G. Diagnostic triage for low back pain: a practical approach for primary care. Med. J. Aust.206 , 268-273 (2017).

2. Bogduk, N. On the definitions and physiology of back pain, referred pain, and radicular pain. Pain 147 , 17-19 (2009).

3. Merskey, H. \& Bogduk, N. IASP taxonomy. Updat. from Pain Terms, A Curr. List with Defin. Notes Usage" (pp 209-214) Classif. Chronic Pain, Second Ed. IASP Task Force Taxon. 209-214 (2012).

4. Bogduk, N. Clinical Anatomy of the Lumbar Spine 85 Sacrum (4th Ed.) . LAVOISIER S.A.S. (2005).

5. El Miedany, Y., Ashour, S., Youssef, S., Mehanna, A. \& Meky, F. A. Clinical diagnosis of carpal tunnel syndrome: Old tests-new concepts.Jt. Bone Spine 75 , 451-457 (2008). 
6. Dellon, A. L. The Four Medial Ankle Tunnels: A Critical Review of Perceptions of Tarsal Tunnel Syndrome and Neuropathy. Neurosurg. Clin. N. Am. 19, 629-648 (2008).

7. Attal, Nadine; Finnerup, N. B. Pharmacological Management of Neuropathic Pain. PAIN Clin. Updat. XVIII , (2010).

8. Finnerup, N. B. et al. Pharmacotherapy for neuropathic pain in adults: a systematic review and metaanalysis. Lancet Neurol.14 , 162-173 (2015).

9. Robertson, K., Marshman, L. A. G. \& Plummer, D. Pregabalin and gabapentin for the treatment of sciatica. J. Clin. Neurosci.26 , 1-7 (2016).

10. Samolsky Dekel, B. G., Sorella, M. C., Vasarri, A. \& Melotti, R. M. Reliability of the Buttock Applied Strain Test to Diagnose Radicular Pain in Patients With Low Back Pain. Pain Pract. papr.12890 (2020). doi:10.1111/papr.12890

11. Freynhagen, R., Baron, R., Gockel, U. \& Tölle, T. R. painDETECT : a new screening questionnaire to identify neuropathic components in patients with back pain. Curr. Med. Res. Opin.22, 1911-1920 (2006).

12. Mathieson, S. \& Lin, C. painDETECT Questionnaire. J. Physiother. 59 , 211 (2013).

13. Freynhagen, R., Tolle, T. R., Gockel, U. \& Baron, R. The painDETECT project - far more than a screening tool on neuropathic pain.Curr. Med. Res. Opin. 32, 1033-1057 (2016).

14. Deyo, R. A. et al. Report of the NIH Task Force on research standards for chronic low back pain. $J$. Pain 15, 569-85 (2014).

15. Farrar, J. T., Young, J. P., LaMoreaux, L., Werth, J. L. \& Poole, R. M. Clinical importance of changes in chronic pain intensity measured on an 11-point numerical pain rating scale. Pain 94, 149-58 (2001).

16. Mulleman, D., Mammou, S., Griffoul, I., Watier, H. \& Goupille, P. Pathophysiology of disk-related sciatica. I.-Evidence supporting a chemical component. Jt. Bone Spine 73 , 151-158 (2006).

17. Olmarker K1, Blomquist J, Stromberg J, Nannmark U, Thomsen P, R. B. Inflammatogenic properties of nucleus pulposus. Spine (Phila Pa 1976). 20, 665-9 (1995).

18. Davis, E. N. \& Chung, K. C. The Tinel Sign: A Historical Perspective. Plast. Reconstr. Surg. 114 , 494-499 (2004).

19. Adachi, S. et al. The tibial nerve compression test for the diagnosis of lumbar spinal canal stenosis-A simple and reliable physical examination for use by primary care physicians. Acta Orthop. Traumatol. Turc. $\mathbf{5 2}, 12-16(2018)$.

20. Costigan, M., Scholz, J. \& Woolf, C. J. Neuropathic Pain: A Maladaptive Response of the Nervous System to Damage. Annu. Rev. Neurosci. 32, 1-32 (2009).

21. JF, H. A neurophysiological basis for the radicular pain of nerve root compression. in Advances in pain research and therapy (ed. Bonica JJ, Liebeskind JC, A.-F. D.) 647-57 (Springer, 1979).

22. Howe, J. F., Loeser, J. D. \& Calvin, W. H. Mechanosensitivity of dorsal root ganglia and chronically injured axons: A physiological basis for the radicular pain of nerve root compression. Pain3 , 25-41 (1977).

23. SMYTH, M. J. \& WRIGHT, V. Sciatica and the intervertebral disc; an experimental study. J. Bone Joint Surg. Am.40 -A , 1401-18 (1958).

24. Shimpo, T., Gilliatt, R. W., Kennett, R. P. \& Allen, P. J. Susceptibility to pressure neuropathy distal to a constricting ligature in the guinea-pig. J. Neurol. Neurosurg. Psychiatry 50 , 1625-1632 (1987).

25. Tinel, J. Le signe du fourmillement dans les lesions des nerfs peripheriques. Press. medicale 47 , 388-389 (1915). 
26. Rebain, R., Baxter, G. D. \& McDonough, S. A systematic review of the passive straight leg raising test as a diagnostic aid for low back pain (1989 to 2000). Spine (2002).

27. Patel, E. A. \& Perloff, M. D. Radicular Pain Syndromes: Cervical, Lumbar, and Spinal Stenosis. Semin. Neurol. (2018). doi:10.1055/s-0038-1673680

28. Finnerup NB1, Sindrup SH, J. T. The evidence for pharmacological treatment of neuropathic pain. Pain $150,573-81(2010)$.

29. Finnerup, N. B. et al. Neuropathic pain: an updated grading system for research and clinical practice. Pain (2016).

30. Tai, Q. et al. Gabapentin In The Treatment Of Neuropathic Pain After Spinal Cord Injury: A Prospective, Randomized, Double-Blind, Crossover Trial. J. Spinal Cord Med. 25 , 100-105 (2002).

31. Yildirim, K. et al. The effectiveness of gabapentin in patients with chronic radiculopathy. Pain Clin. $15,213-218$ (2003).

32. Romano, C. L., Romano, D., Bonora, C. \& Mineo, G. Pregabalin, celecoxib, and their combination for treatment of chronic low-back pain.J. Orthop. Traumatol. (2009). doi:10.1007/s10195-009-0077-z

33. Baron, R. et al. Effectiveness and Safety of Tapentadol Prolonged Release (PR) Versus a Combination of Tapentadol PR and Pregabalin for the Management of Severe, Chronic Low Back Pain With a Neuropathic Component: A Randomized, Double-blind, Phase 3b Study.Pain Pract. (2015). doi:10.1111/papr.12200

34. Baron R, Freynhagen R, Tolle TR, Cloutier C, Leon T, Murphy TK, P. K. A. I. The efficacy and safety of pregabalin in the treatment of neuropathic pain associated with chronic lumbosacral radiculopathy.Pain. 150 , 420-7 (2010).

35. Dworkin, R. H. et al. Recommendations for the Pharmacological Management of Neuropathic Pain: An Overview and Literature Update.Mayo Clin. Proc. 85, S3-S14 (2010).

Table 1. Demographic and clinical variables of the sample and its subsets.

\begin{tabular}{llll}
\hline & Sample & Subsets \\
\hline Variable & $\mathrm{n}(\%)$ & $\begin{array}{l}\text { BUAS+/SLRT- } \\
\mathrm{n}(\%)\end{array}$ & $\begin{array}{l}\text { BUAS+/SLRT+ } \\
\mathrm{n}(\%)\end{array}$ \\
Total & $258(100.0)$ & $155(100.0)$ & $103(100.0)$ \\
Gender & & & \\
Male & $84(32.3)$ & $48(31.0)$ & $36(35)$ \\
Female & $174(67.4)$ & $107(69.0)$ & $67(65)$ \\
Age ${ }^{+}$ & $67.4(15.0)$ & $69.3(14.3)$ & $64.5(15.7)$ \\
Age group (years) & & & \\
A (18-35) & $10(3.8)$ & $6(3.9)$ & $4.0(3.9)$ \\
B (36-50) & $28(10.8)$ & $12(7.7)$ & $16.0(15.5)$ \\
C (51-65) & $58(22.3)$ & $32(20.6)$ & $26.0(25.2)$ \\
D (66-80) & $112(43.1)$ & $70(45.2)$ & $42.0(40.8)$ \\
E (>80) & $50(19.2)$ & $35(22.6)$ & $15.0(14.6)$ \\
Pain site & & & \\
Lumbar spine & $26(10.0)$ & $16(10.3)$ & $10.0(9.7)$ \\
Lumbar spine and Sciatica & $175(67.3)$ & $102(65.8)$ & $73.0(70.9)$ \\
Lumbar spine and diffused pain & $39(14.6)$ & $24(8.4)$ & $6.0(5.8)$ \\
Lumbar spine and other specific pain sites & $19(7.3)$ & $13(15.5)$ & $14.0(13.6)$ \\
\hline
\end{tabular}

+ , mean (SD) years. 
Abbreviations: SLRT, Straight Leg Raising Test (SLRT-, negative SLRT; SLART+, positive SLRT); BUAS, Buttock Applied Strain test (BUAS+, positive BUAS test).

Table 2. Frequency distribution of PD-V1 and PD-V2 outcomes, split by subsets of patients positive for the BUAS test with or without positive SLRT.

\begin{tabular}{lllllll}
\hline Test Group & & & PD- V1 outcome & PD- V1 outcome & PD- V1 outcome & PD- V2 o \\
\hline & & Total n & Positive n (\%) & Uncertain n (\%) & Negative n (\%) & Positive n \\
BUAS + / SLRT - & BUAS + / SLRT - & 155 & $33(21.3)$ & $122(78.7)$ & $0(0.0)$ & $0(0.0)$ \\
BUAS + / SLRT + & BUAS + / SLRT + & 103 & $83(80.6)$ & $20(19.4)$ & $0(0.0)$ & $21(20.4)$ \\
Total & Total & 258 & $116(45,0)$ & $142(55.0)$ & $0(0.0)$ & $21(8.1)$ \\
PD Final score + & PD Final score + & & & & & $0(0.0)$ \\
BUAS + / SLRT - & BUAS + / SLRT - & 155 & $19.0(0.0)$ & $14.6(2.0)$ & $0(0.0)$ & $19.0(0.0)$ \\
BUAS + / SLRT + & BUAS + / SLRT + & 103 & $23.8(2.5)$ & $18.0(0.0)$ & $0(0.0)$ & $19.0(0.0)$ \\
Total & Total & 258 & $22.4(3.6)$ & $15.1(2.2)$ & $0(0.0)$ &
\end{tabular}

+ , Mean $( \pm S D)$

Abbreviations: SLRT, Straight Leg Raising Test (SLRT-, negative SLRT; SLART+, positive SLRT); BUAS, Buttock Applied Strain test (BUAS+, positive BUAS test).

Table 3. Mean scores of BPI items, at V1 and V2, split by subsets of positive BUAS Test with or without positive SLRT.

\begin{tabular}{|c|c|c|c|c|c|}
\hline & BUAS + / SLRT - & BUAS + / SLRT - & BUAS + / SLRT - & BUAS + / SLRT + & BUAS \\
\hline BPI & V1 & $\mathrm{V} 2$ & $\mathrm{~V} 2-\mathrm{V} 1$ & V1 & $\mathrm{V} 2$ \\
\hline Pain intensity & Mean $( \pm \mathrm{SD})$ & Mean $( \pm \mathrm{SD})$ & $\Delta(95 \% \mathrm{CI})^{+}$ & Mean $( \pm \mathrm{SD})$ & Mean \\
\hline 1. Worst Pain & $8.4(1.8)$ & $5.7(1.7)$ & $2.7(2.4-3.1)$ & $8.8(1.4)$ & 5.8( \\
\hline 2. Least Pain & $4.2(2.5)$ & $1.9(1.8)$ & $2.2(1.8-2.6)$ & $4.5(2.6)$ & 1.9( \\
\hline 3. Mean Pain & $6.6(2.0)$ & $3.5(1.7)$ & $3.1(2.7-3.4)$ & $7.0(1.8)$ & 3.5( \\
\hline 4. Actual Pain & $5.5(2.6)$ & $3.1(2.0)$ & $2.4(2.0-2.8)$ & $6.0(2.5)$ & 3.0( \\
\hline 5. Dynamic Pain & $7.9(1.9)$ & $5.1(2.2)$ & $2.9(2.5-3.3)$ & $8.1(2.2)$ & 5.0( \\
\hline 6. Relief with therapy (\%) & $21.0(21)$ & $57.0(23)$ & $36.0(31-40)$ & $20.0(17)$ & 59.0 \\
\hline \multicolumn{6}{|l|}{ Pain interference with } \\
\hline 7. General Activity & $6.3(2.4)$ & $3.7(1.8)$ & $2.6(2.2-3.0)$ & $6.7(2.3)$ & 3.9 \\
\hline 8. Mood & $5.5(2.6)$ & $3.1(2.0)$ & $2.5(2.0-2.9)$ & $6.1(2.7)$ & 3.2( \\
\hline 9. Ambulation & $6.0(2.8)$ & $3.5(2.1)$ & $2.5(2.1-3.0)$ & $6.2(2.9)$ & 3.6( \\
\hline 10. Work & $6.2(2.3)$ & $3.4(1.9)$ & $2.8(2.4-3.2)$ & $6.7(2.4)$ & 3.7 \\
\hline 11. Social interactions & $4.3(2.9)$ & $2.0(2.0)$ & $2.2(1.8-2.7)$ & $4.6(3.0)$ & $2.2(1$ \\
\hline 12. Sleep & $4.5(3.2)$ & $1.9(2.3)$ & $2.6(2.1-3.1)$ & $4.8(3.0)$ & $2.2(2$ \\
\hline 13. Enjoyment of Life & $4.1(2.7)$ & $2.0(2.0)$ & $2.0(1.6-2.5)$ & $4.6(3.0)$ & $2.0(1$ \\
\hline
\end{tabular}

${ }^{+}$, For all BPI Items, and in both BUAS + / SLRT- and BUAS + / SLRT + subsets, V2-V1 differences were significant ( $t$-Test, $p<0.000)$.

Abbreviations: SLRT, Straight Leg Raising Test (SLRT-, negative SLRT; SLART+, positive SLRT); BUAS, Buttock Applied Strain test (BUAS+, positive BUAS test); SD, Standard Deviation; CI, Upper and lower 95\% Confidence Interval.

Table 4. Association analyses between PD-V2 outcomes and independent predictors. 


\begin{tabular}{|c|c|c|c|c|c|}
\hline predictor & & & $\begin{array}{l}\text { Post hoc cell } \\
\text { contribution }\end{array}$ & $\begin{array}{l}\text { Post hoc cell } \\
\text { contribution }+\end{array}$ & $\begin{array}{l}\text { Post hoc cell } \\
\text { contribution }+\end{array}$ \\
\hline & $\chi^{2}$ & p Value & $\begin{array}{l}\text { PD-V2 } \\
\text { Negative }\end{array}$ & Uncertain & Positive \\
\hline Gender & 1.012 & $>0.05$ & & & \\
\hline Age group & 6.433 & $>0.05$ & & & \\
\hline Pain site & 2.162 & $>0.05$ & & & \\
\hline SLRT-V1 & 115.110 & $<0.0001$ & & & \\
\hline Positive & & & & 8.189 & \\
\hline Negative & & & 10.729 & & \\
\hline PD-V1 & 93.637 & $<0.0001$ & & & \\
\hline Positive & & & & 7.386 & \\
\hline Uncertain & & & 9.677 & & \\
\hline
\end{tabular}

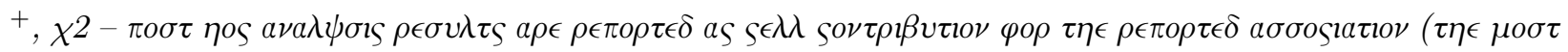
$\imath \nu \varphi \lambda v \epsilon \nu \tau \tau \omega o)$.

Abbreviations: SLRT, Straight Leg Raising Test; PD, painDetect questionnaire; V1, first visit; V2, follow up visit.

Table 5. Multinomial logistic regression, significant results.

\begin{tabular}{|c|c|c|c|c|c|c|}
\hline & Effect / Predictor & $\chi^{2}$ & $\mathrm{DF}$ & $\mathrm{p}$ & Adjusted Odds Ratio & Adjusted Odds Ratio \\
\hline & & & & & $\mathrm{n}$ & $\begin{array}{l}95 \% \text { CI } \\
\text { Lower }\end{array}$ \\
\hline Model fitting (final) & Model fitting (final) & 130.097 & 6 & 0.000 & & \\
\hline Likelihood ratio & $\begin{array}{l}\text { Likelihood ratio } \\
\text { SLRT-V1 outcomes }\end{array}$ & 147.826 & 3 & 0.000 & & \\
\hline $\begin{array}{l}\text { Parameter estimates } \\
\text { SLRT Negative }\end{array}$ & $\begin{array}{l}\text { Parameter estimates } \\
\text { PD-V2 Positive } \\
\text { PD-V2 Uncertain }\end{array}$ & & & $\begin{array}{l}0.000 \\
0.000\end{array}$ & $\begin{array}{l}0.038 \\
0.038\end{array}$ & $\begin{array}{l}0.012 \\
0.016\end{array}$ \\
\hline
\end{tabular}

CI, 95\% confidential intervals of the Adjusted Odds Ratio.

+, Comparison outcomes (V1-SLRT, positive and negative outcomes) versus reference outcome (V2-PD, negative outcome) as yielded by the multinomial logistic regression model.

++ , with regard to the predictor, the risk of the considered comparison outcomes to occur versus the reference outcome (PD, negative) decreases when the adjusted odds ratio (and 95\% CI lower and upper values) are $<1$ and increases when the adjusted odds ratio (and 95\% CI lower and upper values) values are $>1$, respectively.

Figures legends.

Fig. 1. Flowchart of the study.

Foot notes:

LBP, Low Back Pain,

RP, Radicular Pain,

BUAS-test, Buttock Applied Strain test,

SLRT, Straight Leg Raising Test, 
V1, first visit.

V2, follow-up visit.

BPI, Brief Pain Inventory,

PD, PainDETECT questionnaire.

Fig. 2. Mean scores of the BPI items in the sample, at V1 and V2, and their V2-V1 absolute differences.

Foot notes:

For BPI items 1-13 names, see table 3.Solid line, absolute differences of BPI items' mean score between V1 and V2; values are anchored by a dashed line.For all BPI-items, the V2-V1 differences were statistically significant, (t-test, $p<0.001$, respectively). 


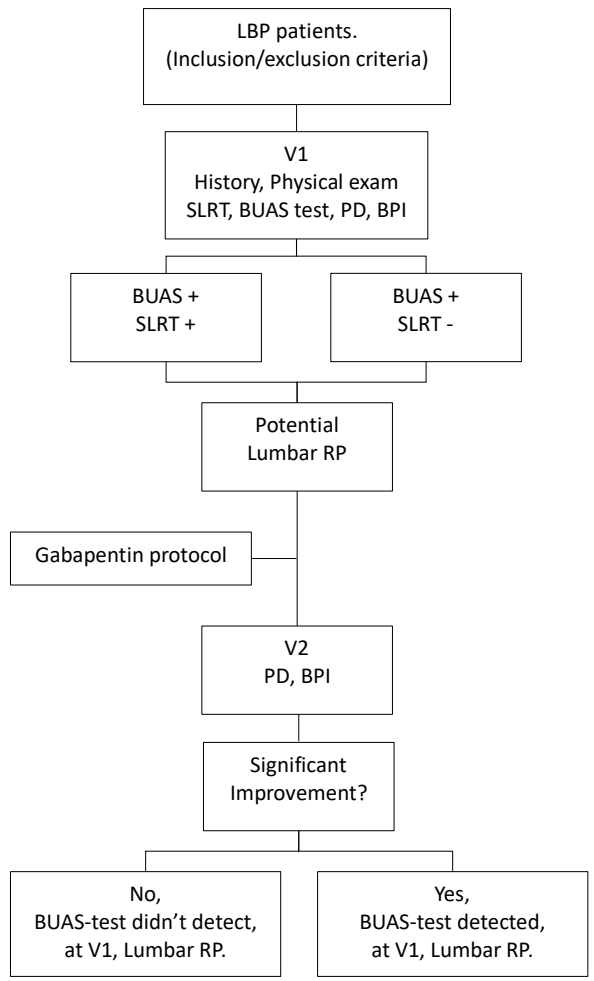




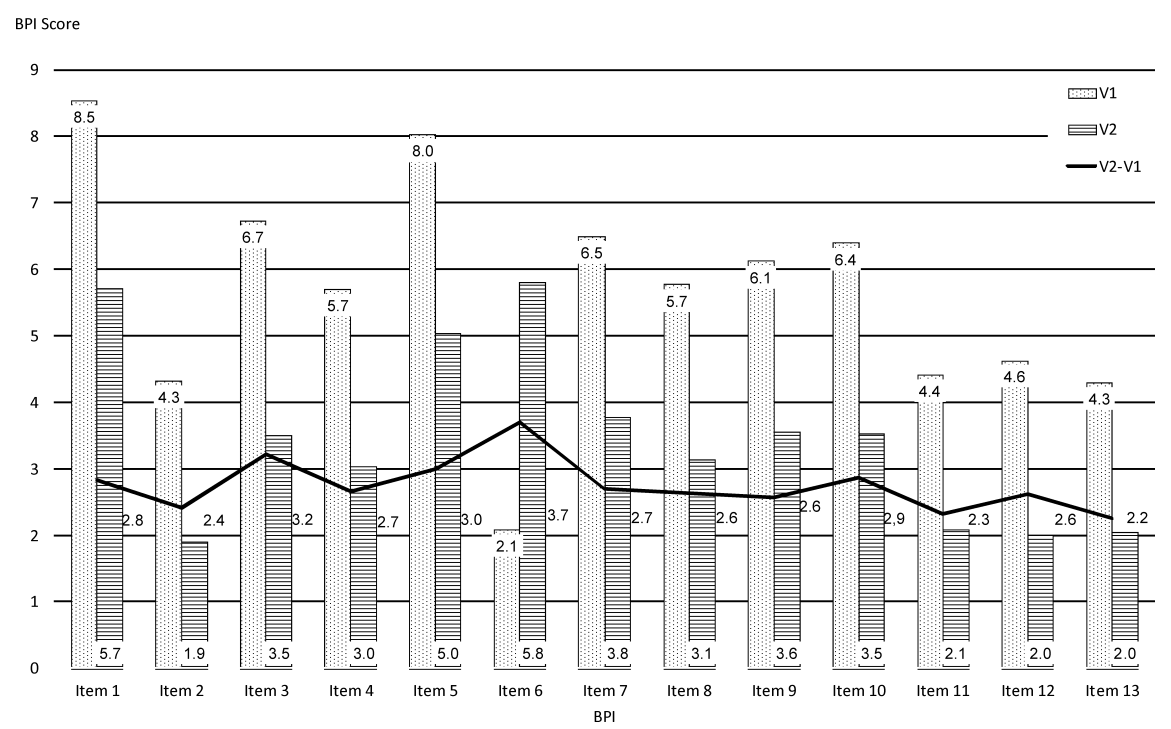

\section{Hosted file}

Ex ADIUV tab 1.docx available at https://authorea.com/users/318718/articles/448631-evidencefor-the-buas-test-ability-to-diagnose-lumbar-radicular-pain-in-low-back-pain-patients

\section{Hosted file}

Ex ADIUV tab 2 new.docx available at https://authorea.com/users/318718/articles/448631evidence-for-the-buas-test-ability-to-diagnose-lumbar-radicular-pain-in-low-back-painpatients

\section{Hosted file}

Ex ADIUV tab 3 solo BUAS CI ok senza p.docx available at https://authorea.com/users/318718/ articles/448631-evidence-for-the-buas-test-ability-to-diagnose-lumbar-radicular-pain-inlow-back-pain-patients

\section{Hosted file}

EX ADIUV tab 4 tab qhi OK.docx available at https://authorea.com/users/318718/articles/ 448631-evidence-for-the-buas-test-ability-to-diagnose-lumbar-radicular-pain-in-low-backpain-patients

\section{Hosted file}

Ex ADIUV tab 5 MLM ok.docx available at https://authorea.com/users/318718/articles/448631evidence-for-the-buas-test-ability-to-diagnose-lumbar-radicular-pain-in-low-back-painpatients 